Ann. Biol. anim. Bioch. Biophys., I975, 15 (I), 29-36.

\title{
UTILISATION D'IMPLANTS PROGESTAGÈNES SOUS CUTANÉS POUR LA MAÎTRISE DES CYGLES CHEZ LES VACHES ALLAITANTES DE RACE SALERS. DÉTERMINATION DE LA DOSE ET DE LA DURÉE DE SÉJOUR OPTIMA
}

\author{
J. PELOT, J. P. OLIVIER* et D. CHUPIN \\ Station de Physiologie de la reproduction, \\ Centre de Recherches de Tours, I. N. R. A., \\ B. P. 1, Nouzilly, 37380 Monnaie \\ * E. D. E. du Cantal, \\ 15000 Aurillac
}

RÉSUMÉ

L'efficacité des implants sous-cutanés de SC 2 roog (GD Searle et Co) pour maîtriser l'œestrus chez la vache allaitante de race Salers est améliorée par une diminution de la durée de séjour et une augmentation de la dose de progestagène. Ainsi, le pourcentage maximum de femelles en cestrus sur une période de $36 \mathrm{~h}$ passe de $58,9 \mathrm{p}$. roo pour un traitement de II jours à la dose de $6 \mathrm{mg}$ à 85,9 p. Ioo pour un traitement de $9 \mathrm{j}$ à la dose de $\mathrm{I} 2 \mathrm{mg}$. De même, pour ces deux traitements les taux de femelles mettant bas après insémination artificielle lors de l'œstrus induit détecté sont de 40,3 p. I00 et 64,7 p. roo $(\mathrm{P}<0,0 \mathrm{I})$.

Avec le traitement d'une durée de 9 jours avec une dose de $12 \mathrm{mg}$ de SC 21009 deux inséminations artificielles sont possibles et on a obtenu sur un effectif important ( 320 vaches) 60,3 p. roo des vaches mettant bas à la suite de ces deux interventions systématiques.

Le taux de femelles non gestantes en fin de compagne de reproduction est de 5,o p. 100 .

Le taux cumulé de mises bas sur une période de trente jours reste faible (70 p. Ioo) du fait de difficultés de détection des retours en cestrus (stabulation entravée). Des traitements systématiques de ces femelles non gestantes après l'œstrus induit peuvent être proposés.

La très grande activité biologique d'un dérivé de la I9-nor-progestérone, le SC 2 roog (Searle et Co.), permet la réalisation d'implants de petite taille pour le contrôle du cycle œstrien chez la Vache. Les premiers résultats ont montré l'importance de la dose de progestagène, de la durée de séjour de l'implant et de l'injection en début de traitement de valérate d'œstradiol et en fin de traitement de PMSG (Chupin et al., I974 a). Cette étude précise les taux de fertilité obtenus sur une popu- 
lation de 976 vaches Salers allaitantes selon les modalités d'emploi de ce traitement pendant deux années consécutives.

L'étude a permis la comparaison de deux durées de traitement (9 et II jours), de trois doses $(6,9$ et $\mathrm{I} 2 \mathrm{mg})$ et de l'importance du moment de dépôt de la semence par rapport au moment de l'ovulation en comparant la fertilité après détection de l'œstrus ou après deux inséminations à des moments prédéterminés après le retrait des implants.

\section{MATÉRIEL ET MÉTHODES}

Les vaches appartenaient à des éleveurs du Cantal. Leur degré de parité était variable, s'étendant de I à I I. Elles ont toutes été traitées entre février et avril.

La répartition des animaux selon les combinaisons expérimentales est donnée dans le tableau $\mathrm{I}$.

TABLEAU I

Matériel et méthodes. Répartition des effectifs

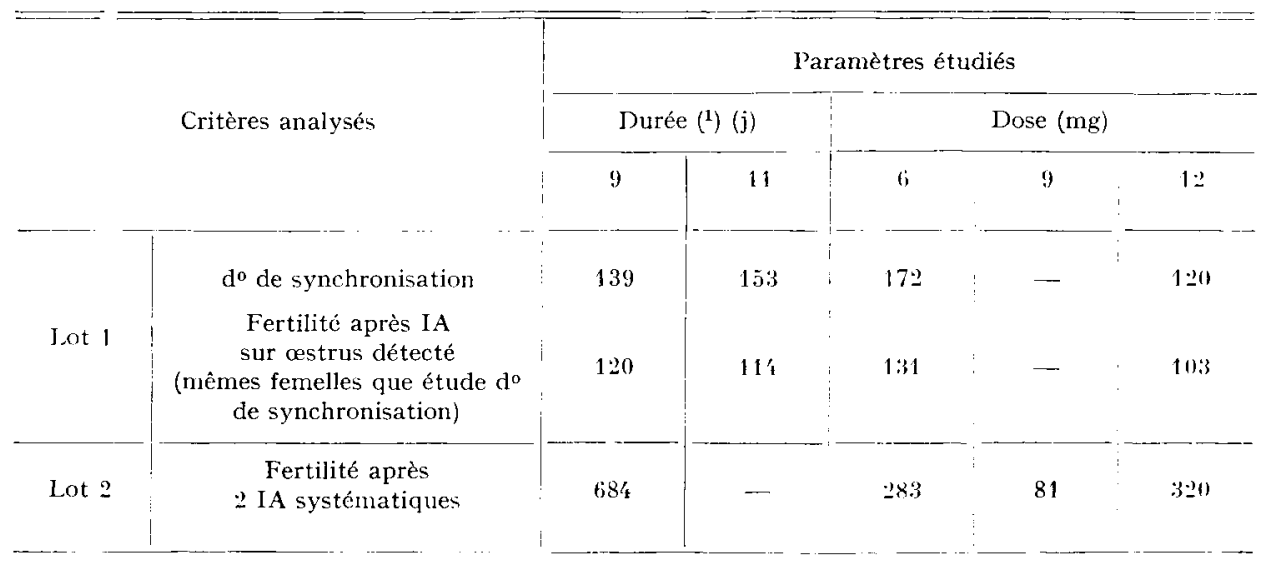

(1) Jour de la pose $=\mathrm{J}_{0}$.

Remarques: Dans nos études précédentes (Chuprs et al., 197ł a) le jour de la pose était $\mathrm{J}_{1}$ et les durées étudiées ici avaient été dénombrées 10 et 12 jours.

Les inséminations ont été effectuées de la façon suivante :

Lot 1 : L'observation du comportement des femelles entre elles servait à détecter l'œestrus deux fois par jour lors de leurs mises en liberté. Les animaux ainsi détectés ont été inséminés deux fois I $2 \mathrm{~h}$ et $24 \mathrm{~h}$ après la première observation avec la semence d'un seul taureau de race Charolaise (7 I p. Ioo non retours, 60-90 jours):

Les femelles n'ayant pas manifesté d'oestrus 3 jours après le retrait des implants ont été inséminées systématiquement le $4^{\mathrm{e}}$ jour matin et soir (approximativement 96 et 108 h après le retrait des implants).

Lot 2 : Toutes les femelles ont été inséminées systématiquement $48 \mathrm{~h}$ et $7^{2} \mathrm{~h}$ après le retrait des implants. Plusieurs taureaux de race Charolaise et Salers ont été utilisés..

Les modalités pratiques d'utilisation des implants ainsi que la méthodologie de déponillement des résultats de fertilité ont été décrites précédemment (CHuPIN et al., I974 $a$ ). 
L'efficacité de la synchronisation des cestrus a été exprimée ici par plusieurs critères :

- l'étendue, qui fournit de plus un renseignement sur l'efficacité du blocage (les premières venues en cestrus précoces, I 2 ou $24 \mathrm{~h}$ après le retrait des implants, indiquent une inhibition limite) ;

- le mode : avec les progestagènes, il est généralement à $48 \mathrm{~h}$ sauf en cas de blocage insuffisant $(36 \mathrm{~h}$ voire $24 \mathrm{~h})$;

- le pourcentage d'animaux en œstrus entre 36 et $72 \mathrm{~h}$ après le retrait des implants; ce critère donne une estimation des possibilités d'inséminations artificielles systématiques ;

- le pourcentage d'animaux non observés en oestrus en I $44 \mathrm{~h}$ qui regroupent les animaux non synchronisés et les erreurs de détection d'œestrus. La distinction entre ces deux cas peut être faite lorsque l'on a une observation d'oestrus 3 semaines plus tard.

\section{RÉSULTATS}

Les résultats ont été analysés en fonction du degré de synchronisation et du taux de fertilité.

\section{I. - Le degré de synchronisation (lot I)}

Les quatre critères analysés ont été rassemblés dans le tableau 2. Pour les deux doses, le traitement d'une durée de 9 jours donne une meilleure synchronisation (étendue, pourcentage en œstrus entre 36 et $72 \mathrm{~h}$, pourcentage non observé en œstrus en $\mathrm{I} 44 \mathrm{~h}$ ), mais la différence n'est significative que pour la dose de $6 \mathrm{mg}: 58,9 \mathrm{p}$. Ioo des femelles sont en œstrus entre 36 et $7^{2} \mathrm{~h}$ après un traitement de II jours contre 82,9 . p. Ioo après un traitement de 9 jours. De même, pour ces deux durées de traitement et toujours pour une dose de $6 \mathrm{mg}$, les taux de femelles non observées en œstrus en $\mathrm{I} 44 \mathrm{~h}$ sont de $3 \mathrm{I}, \mathrm{I} \mathrm{p}$. Ioo et $\mathrm{I} 3,7 \mathrm{p}$. Ioo.

\section{TABLEAU 2}

Synchronisation de l'ostrus après le retrait d'implants sous-cutanés de SC 21009 che r la vache allaitante de race Salers

Influence de la dose de progestérone et de la durée de séjour

\begin{tabular}{|c|c|c|c|c|c|c|}
\hline \multicolumn{2}{|c|}{ Traitement } & \multirow{2}{*}{$\begin{array}{c}\text { Eiffectifs } \\
52 \\
57\end{array}$} & \multirow{2}{*}{$\begin{array}{c}\begin{array}{c}\text { Etendue } \\
\text { des venues }\end{array} \\
\text { en œstrus (h) } \\
\begin{array}{c}24-72 \\
24-72\end{array}\end{array}$} & \multirow{2}{*}{$\begin{array}{c}\text { Mode }(h) \\
48 \\
48\end{array}$} & \multirow{2}{*}{ 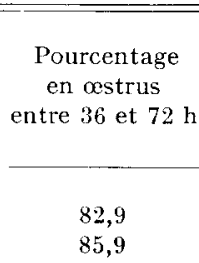 } & \multirow{2}{*}{$\begin{array}{c}\text { Pourcentage } \\
\text { non vu en } \\
\text { ostrus en 14' h } \\
\\
13,7 \\
7,0\end{array}$} \\
\hline 9 jours & $\begin{array}{r}6 \mathrm{mg} \\
12 \mathrm{mg}\end{array}$ & & & & & \\
\hline 11 jours & $\begin{array}{r}6 \mathrm{mg} \\
12 \mathrm{mg}\end{array}$ & $\begin{array}{l}90 \\
36\end{array}$ & $\begin{array}{l}24-144 \\
12-84\end{array}$ & $\begin{array}{l}36 \\
60\end{array}$ & $\begin{array}{l}58,9 \\
82,5\end{array}$ & $\begin{array}{c}31,1 \\
14,3\end{array}$ \\
\hline
\end{tabular}

\section{2. -- Le taux de fertilité après IA sur aestrus observé (lot I)}

Il est exprimé par le taux de mises bas consécutives aux inséminations artificielles à l'œstrus induit, par le taux de mises bas dans une période de 30 jours selon la méthodologie définie par Chupin et al. (I974 a) (tabl. 3) et par le taux d'animaux vides (tabl. 4). 
TABLEAU 3

Fertilité à l'astrus induit (exprimée en taux de mise bas) et taux mise bas de en 30 jours. Infuence de la dose initiale et de la durée de séjour de l'implant

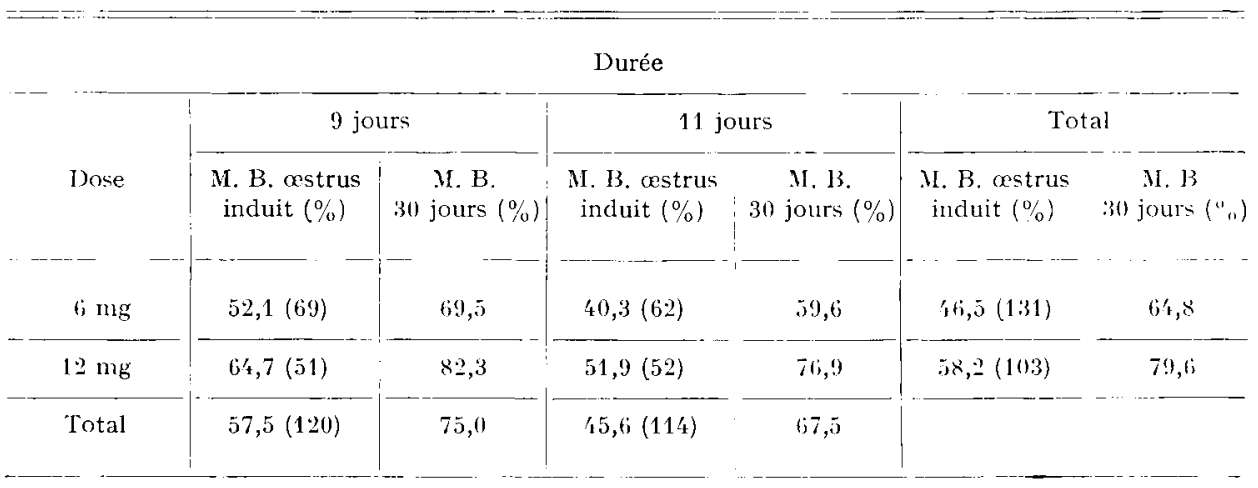

( ) Nombre d'animaux traités.

TABIEAU 4

Pourcentage d'animaux non fécondés en fin de campagne de reproduction (lot I)

\begin{tabular}{|c|c|c|}
\hline \multirow{2}{*}{ Dose } & \multicolumn{2}{|c|}{ Durée } \\
\hline & 9 jours $(\%)$ & 11 jours $\left(\begin{array}{l}0 \\
0\end{array}\right)$ \\
\hline $6 \mathrm{mg}$ & $5,7(69)$ & $11, ;(6 ; 2)$ \\
\hline $12 \mathrm{mg}$ & $0 \quad(51)$ & $7,7(5.3)$ \\
\hline
\end{tabular}

Les résultats portés dans ces tableaux 3 et 4 ne concernent que les animaux inséminés après observation d'œstrus. Les femelles non observées en œstrus et inséminées systématiquement 96 et Io $8 \mathrm{~h}$ après le retrait des implants ont un taux de fécondation très faible $(20,4$ p. I0o), ces inséminations ayant été réalisées beaucoup trop tardivement s'il s'agit d'ovulations silencieuses.

On observe une tendance (non significative) à une augmentation de la fertilité en liaison avec une diminution de la durée de séjour et une augmentation de la dose.

Cependant, si on compare les traitements extrêmes (9 jours, I 2 mg et I I jours, $6 \mathrm{mg})$, on observe une différence significative $(\mathrm{P}<0, \mathrm{OI})$ pour les deux paramètres de fertilité : taux de mises bas consécutives aux IA à l'œestrus induit $(64,7 \mathrm{p}$. Ioo vs 40,3 p. Ioo respectivement) et taux de mises bas en 30 jours (82,3 p. Ioo vs 59,6 p. roo).

Les pourcentages d'animaux vides en fonction de la dose et de la durée du traitement sont portés dans le tableau 4. 
Comme précédemment, on observe une différence significative $(\mathrm{P}<0, \mathrm{OI})$ du taux d'animaux vides entre les traitements 9 jours $12 \mathrm{mg}$ et II jours $6 \mathrm{mg}$ (o p. IOO vS II,3 p. roo).

\section{3. - Le taux de fertilité après IA systématiques (durée 9 jours)}

Les mêmes paramètres que pour le paragraphe précédent ont été analysés dans le tableau 5. On observe une élévation de la fertilité à l'œstrus induit de 43,4 à $60,3 \mathrm{p}$. Ioo lorsque la dose initiale de progestagène augmente de 6 à $\mathrm{I} 2 \mathrm{mg}$ ( $\mathrm{P}<0,0 \mathrm{I})$. Le taux de mises bas en 30 jours varie moins nettement $(6 \mathrm{I}, 4 \mathrm{p}$. Ioo is $69,0 \mathrm{p}$. Ioo lorsque la dose croît de 6 à I $2 \mathrm{mg}$ ) (NS). Enfin, le taux de femelles non fécondées en fin de campagne de reproduction est de $3,5 \mathrm{p}$. Ioo avec la dose de $6 \mathrm{mg}$ et $6,4 \mathrm{p}$. Ioo avec la dose de $12 \mathrm{mg}$ (NS) avec une valeur moyenne de 5,0 p. IOO. Les implants $9 \mathrm{mg}$ donnent des résultats intermédiaires pour tous les critères $(55,5 \mathrm{p}$. roo de mises bas consécutives à l'ostrus induit).

TABLEAU 5

Fertilité après deux IA systématiques à l'oestrus induit, taux de mises bas en 30 jours et taux d'animaux non fécondés(lot 2)

\begin{tabular}{|c|c|c|c|c|}
\hline 1)ose (mg) & Effectifs & $\begin{array}{l}\text { Pourcentage } \\
\text { M. B. } \\
\text { ostrus induit }\end{array}$ & $\begin{array}{c}\text { Pourcentage } \\
\text { M. B. } \\
\text { en } 30 \text { jours }\end{array}$ & $\begin{array}{l}\text { Pourcentage } \\
\text { de femelles } \\
\text { non fécondées }\end{array}$ \\
\hline 6 & 283 & 43,4 & $61,{ }^{\prime}$ & 3,5 \\
\hline 9 & 81 & 55,5 & 70,3 & 4,7 \\
\hline 12 & 320 & 60,3 & 69,0 & 6,4 \\
\hline Total & 681 & 52,8 & 66,0 & 5,0 \\
\hline
\end{tabular}

\section{DISCUSSION}

Pour tous les critères étudiés (synchronisation, fertilité à l'œstrus induit, taux de mises bas en 30 jours, taux d'animaux non fécondés), les meilleurs résultats sont obtenus avec le traitement d'une durée de 9 jours avec une dose de $12 \mathrm{mg}$ de SC 2 Ioog.

A l'opposé, les résultats les plus faibles sont obtenus avec le traitement d'une durée de I I jours avec une dose de $6 \mathrm{mg}$ de SC 2 roog.

La plus courte durée de traitement et la plus forte dose de SC 2 Ioog se traduisent par une amélioration pour tous les critères.

Les résultats de WILTBANK et KASSON (I968) avaient montré l'intérêt des traitements courts ( 9 jours) comparés à des traitements plus longs ( 6 jours). Dans une étude précédente (CHUPIN et al., I974 a), nous avons comparé des traitements d'une durée de 9, II, I3, I5 ou I7 jours et conclu à l'intérêt des traitements de 9 ou II jours sur les traitements plus longs. 
Le principe de ces traitements d'une durée inférieure à celle du cycle cestrien repose sur l'utilisation en début de traitement du valérate d'œstradiol comme facteur lutéolytique. Nous avons montré précédemment que lorsqu'un traitement de ce type est réalisé sur des femelles cycliques en utilisant la noréthandrolone comme progestatif, les venues en œstrus après l'arrêt du traitement sont très dispersées (Chupin et al., I974 $b$; MAULÉon, I973). Les taux de progestérone plasmatique au cours d'un tel traitement n'évoluent pas différemment (HANSEL, I972)ou peu différemment (raccourcissement de 2 jours : M. LEMON, communication personnelle) de ce que l'on peut observer lors d'un cycle normal. Les femelles traitées entre $\mathrm{J}_{0}$ et $\mathrm{J}_{6}$ conservent leur cycle.

Pourtant, les résultats présentés dans le tableau 2 font état d'une synchronisation efficace ( $86 \mathrm{p}$. Ioo en $36 \mathrm{~h}, 7 \mathrm{p}$. Ioo des animaux non observés en nestrus) bien qu'il s'agisse d'un traitement d'une durée de 9 jours.

La majorité des femelles traitées sont en anœstrus sans présence d'un corps jaune (70 à 80 p. roo n'ont pas de progestérone détectable : THIMONIER et al., à paraître). L'utilisation de valérate d'œestradiol ne vise donc pas à provoquer une lutéolyse mais à renforcer l'effet du progestatif sur le freinage de l'exécution des gonadotrophines. On sait en effet que la rétroaction négative de l'œstradiol est renforcée par un progestagène.

On sait également que l'œestradiol et la progestérone respectivement sensibilisent et désensibilisent l'hypophyse aux " releasing factors " hypothalamiques. La présence prolongée d'œstradiol due à l'action retard du valérate d'œstradiol peut donc faciliter la réponse de l'hypophyse aux facteurs de décharge à l'arrêt du traitement.

Enfin, le valérate d'œstradiol peut exercer son action par le biais d'une meilleure préparation du tractus génital à la fécondation et notamment à la capacitation des spermatozoïdes.

Le traitement d'animaux non cycliques peut être considéré comme une mise en charge de l'hypophyse permettant la première décharge cyclique après l'arrêt du traitement selon un schéma identique à celui des traitements utilisés chez la femme aménorrhéique.

La durée optimum de traitement représente un compromis entre cette mise en charge de 1'hypophyse et les effets défavorables d'une imprégnation prolongée de progestagène. Les résultats présentés ici montrent qu'elle est inférieure ou égale à 9 jours pour les vaches allaitantes de race Salers.

Cet effet de mise en charge de l'hypophyse suppose que l'inhibition de la libération soit efficace pendant tout le traitement. La relative inefficacité d'une dose de 6 mg jugée d'après le groupage des venues en œstrus ainsi que la fertilité plus faible, malgré des inséminations réalisées sur chaleurs observées (lot I), pourrait traduire une fuite d'hormones gonadotropes hypophysaires en fin de traitement. Les décharges ( $\mathrm{LH}$ notamment) seraient alors quantitativement insuffisantes, ou décalées chronologiquement.

Des résultats similaires ont été obtenus sur la brebis (PELIETIER et CogNiE, à paraître) : le pic de $\mathrm{LH}$ obtenu après un traitement de $\mathrm{I} 2$ jours est inférieur (niveau maximum et durée) à celui obtenu après un traitement de 6 jours chez la brebis allaitante à contre saison. Toutefois, les taux de fertilité obtenus avec deux doses de FGA (20 ou $40 \mathrm{mg}$ ) en éponge vaginale laissée en place pendant 6 ou I2 jours 
ne sont pas significativement différents (CoGNIE, communication personnelle). Après deux IA systématiques (lot 2) à intervalles fixés par rapport au retrait des implants, la fertilité à l'œstrus induit est élevée $(60,3$ p. Ioo) pour le traitement qui induit une bonne synchronisation (9 jours, I2 $\mathrm{mg}$ ). Par contre, avec une dose plus faible $(6 \mathrm{mg})$, la baisse de fertilité notée en inséminant avec détection d'ostrus est accentuée en IA systématique par un étalement plus grand des venues en ostrus $(43,4$ p. Ioo avec IA systématiques contre $52, \mathrm{I}$ p. Ioo en inséminant avec détection d'œestrus).

Les taux de mises bas en 30 jours (lot 2) sont faibles compte tenu de la fertilité de l'œstrus : pour roo femelles non fécondées à l'œstrus induit, 27 seulement le sont dans le mois qui suit. Ceci s'explique par les conditions de conduite des animaux au moment des traitements (stabulation entravée, période hivernale). Ce n'est que lors de la mise à l'herbe (mi-mai alors que les traitements commencent fin janvier) que ces femelles ont réellement la possibilité d'être fécondées.

Le taux de femelles non fécondées en fin de campagne $(5,0 \mathrm{p}$. Ioo) est inférieur à celui publié pour la race Salers $(8, \mathbf{I}-\mathbf{I 0}, 7$ p. IOo, E. D. E. du Cantal).

Chez la vache Salers allaitante, un traitement de 9 jours avec r $2 \mathrm{mg}$ de SC 21009 en implants sous-cutanés induit un œstrus d'un fertilité normale $(60,3$ p. Ioo de mises bas après 2 IA systématiques). Des améliorations peuvent être apportées ; elles concernent les conditions pratiques d'utilisation (durée, dose), mais des progrès du taux de fertilité à l'œstrus induit nécessitent de nouveaux progrès de l'insémination artificielle elle-même, en particulier par un meilleur tri des éjaculats selon leur qualité.

Pour la fécondation des retours en ostrus par contre, une recherche des moyens de détection d'œstrus en stabulation entravée et/ou des possibilités de retraitements systématiques des femelles non fécondées à l'œstrus induit est nécessaire avant la mise à 1'herbe. Un schéma peut être proposé utilisant conjointement diagnostic précoce de gestation et analogues des prostaglandines qui permettrait une insémination artificielle systématique 30 jours après l'œstrus induit.

Reçu pour publication en janvier 1975.

\section{REMERCIEMEN'TS}

Nous tenons à remercier Ie Dr F. LE Provost (Searle Europe) pour la fourniture des produits et les conseils quant à leur utilisation, ainsi que tous les éleveurs qui nous ont prêté leur concours.

\section{SUMMARY}

USE OF SUBCUTANEOUS PROGESTAGEN IMPLANTS

FOR CONTROL OF THE CYCLE IN SALERS SUCKLING COWS. DETERMINATION OF DOSE AND OPTIMAL PERIOD OF DURATION

The efficiency of subcutaneous implants of SC 2 roog (Searle and Co.) for estrus control of the Salers suckling cow is improved by shortening the period of duration of the implant and increasing the progestagen dose. Thus, the maximum percentage of females in estrus in a 36 -hour period 
rises from $58.9 \mathrm{p}$. Ioo for an I I-day treatment at a dose of $6 \mathrm{mg}$ to $85.9 \mathrm{p}$. Ioo for a 9-day treatment at a dose of $12 \mathrm{mg}$. Moreover, the percentages of females calving after artificial insemination following detected induced estrus are $40.3 \mathrm{p}$. Ioo and $64.7 \mathrm{p}$. IOO $(\mathrm{P}<0.0 \mathrm{r})$, respectively, for the two treatments.

Two artificial inseminations can be done with the 9-day treatment using a $12 \mathrm{mg}$ dose of $\mathrm{SC} 21009 ; 60.3$ p. Ioo of 320 cows inseminated this way, calved at the end of the two systematic treatments. The percentage of non-pregnant females at the end of the reproduction period is 5.0 p. Ioo.

The cumulative calving rate over a 30 -day period remains low (7o p. Ioo) due to difficulty in detecting returns to estrus (tying stalls). Systematic treatment of these non-pregnant females after induced estrus is suggested.

\section{RÉFÉRENCES BIBLIOGRAPHIQUES}

Chupir D., Deletang F., Petit M., Pelot J., Le Provost F., Ortavant R., Parez M., Mauléon P., I974a. Utilisation de progestagènes en implants sous-cutanés pour la maîtrise des cycles sexuels chez les bovins. Ann. Biol. anim. Bioch. Biophys., 14, 27-39.

Chupin D., Pelot J., Mauléon P., r974 $b$. Comparaison des taux de conception obtenus après inséminations artificielles au premier ou au second ostrus après des traitements de synchronisation par la noréthandrolone chez la Vache. Ann. Biol. anim. Bioch. Biophys., 14, 2 I-26.

Cognie Y., I974. (Communication personnelle).

Hansel W., Schechter R. E., 1972. Biotechnical procedures for control of the ostrous cycle of domestic animals. VII ${ }^{\mathrm{e}}$ Cong. intern. Reprod. anim. Insem. artif., Munich, vol. I, 78-g6.

Lemox M., I973. (Communication personnelle).

Mavléox P., r973. New trends in the control of reproduction in the bovine. Cong. Fédér. Furop. Zootech., Vienne, Sept. 1973. Livestock Production Science, 1974, 1, II7-1 3 I.

Pelletier J., Cognie Y., cités par Pelletier J., Thimonier J., i975. Interaction entre les stéroïdes et la décharge de LH. Ann. Biol. anim. Bioch. Biophys., (in press).

Thimoniek J., Pelot J., Chupin D., r975. Estimation de l'activité ovarienne post-partum par dosaye de la progestérone plasmatique chez la Vache Salers allaitante (a paraître).

Wiltbaxk J. N., Kasson C. W., I968. Synchronization of oestrus in cattle with an oral progestational agent and an injection of an oestrogen. J. Anim. Sci., 29, I×3-II6. 